\title{
Recurrent Pancreatitis in the Setting of Superior Mesenteric Artery Syndrome
}

\author{
Rajshri Joshi ${ }^{1}$, Jafar Alzubi ${ }^{2}$, Mo'tasem Alkhayyat ${ }^{3}$, Amar Gupta ${ }^{2}$, and Daniel Chapa ${ }^{2}$ \\ ${ }^{1}$ Ohio University Heritage College of Osteopathic Medicine Cleveland Campus \\ ${ }^{2}$ Cleveland Clinic \\ ${ }^{3}$ Cleveland Clinic Foundation
}

September 14, 2020

\begin{abstract}
Various causes of Recurrent Acute pancreatitis have been identified in the past. This report presents a novel case of "recurrent" acute pancreatitis in the setting of superior mesenteric artery syndrome (SMAS). Thus, highlighting SMAS as a rare yet possible cause of recurrent pancreatitis facilitating early diagnosis and prompt treatment.
\end{abstract}

\section{Introduction}

Superior mesenteric artery syndrome (SMAS) is a clinical condition that is characterized by compression of the third part of duodenum between the aorta posteriorly and the superior mesenteric artery (SMA) anteriorly. Von Rokitansky described the syndrome for the first time in 1861. Later, in 1927, pathophysiology of SMAS was described in detail by Wilkie, giving this syndrome another name "Wilkie's syndrome". ${ }^{1}$ This syndrome can present with very non-specific symptoms and thus making it difficult for prompt diagnosis and differentiating it from other conditions such as cholelithiasis, peptic ulcer disease, and abdominal angina. Therefore, the key to diagnose this condition is a high index of suspicion. In this report, we present an unusual yet possible case of "recurrent" acute pancreatitis as a result of superior mesenteric artery syndrome.

\section{Case presentation}

A 45-year-old man presented to the hospital with a 24-hour history of worsening upper abdominal, bilateral flank pain and nausea without emesis. His past medical history consists of congestive heart failure, history of acute myocardial infarction, lower urinary tract symptoms, benign prostatic hyperplasia, vitiligo, congenital utero pelvic junction obstruction status post left pyeloplasty. He experienced similar symptoms months back when it resolved on its own. On presentation, he was hemodynamically stable with history significant for unintentional weight loss of 35Ibs. The patient denied fever, chills or loose stools. Physical examination was significant for epigastric tenderness. Laboratory data demonstrated normal kidney function, glucose 100 (70-99 mg/dL), lipase 1552 (73-393U/L), unremarkable urinalysis, albumin 3.1 (3.4-5.4 g/dL) and calcium $8.3(8.6-10.3 \mathrm{mg} / \mathrm{dL})$. Diagnostic imaging included contrasted-computed tomography (CT) of the abdomen and pelvis, which showed inflammation in the tail of pancreas without necrosis. In addition, dilation of the second and third portion of the duodenum with an abrupt change in caliber (Figures 1-2) along with narrow aortomesenteric angle of $18^{\circ}$ (Figure 3) was also observed. The patient was managed conservatively with intravenous fluids, intravenous morphine and nothing per mouth status. Gastric decompression was not performed because of incomplete obstruction. Diet was advanced gradually. Other potential causes of acute pancreatitis like gall bladder stones or biliary obstruction, alcohol or drug induced pancreatitis were ruled out. Ultrasound showed no gall stones or biliary obstruction. Based on the patient's clinical presentation, laboratory and radiographic findings, he was diagnosed with SMAS -associated acute pancreatitis. Patient 
was readmitted again two months later with a similar presentation. CT of the abdomen and pelvis showed acute pancreatitis complicated by splenic vein thrombosis with resolution of duodenal dilation. During the recurrent episode, he was again conservatively treated as well with subsequent excellent recovery.

\section{Discussion}

SMAS is considered a rare but serious condition with an estimated incidence of $0.1-0.3 \% .^{2}$ Though SMAS can occur at any age, it has been mostly reported in adolescent or young adults. Anatomically, SMA rises from the aorta at L1-2 level forming an angle with the aorta called aortomesenteric angle, normally $45-60^{\circ}$, while the third part of duodenum passes between the aorta and SMA at L3 level. ${ }^{3}$ Normally, a retroperitoneal fat pad sitting at the angle between the aorta and SMA maintains adequate aortomesenteric angle, and thus prevents duodenal compression. ${ }^{3,4}$ Hence, factors that result in a loss of retroperitoneal fat and a decrease of aortomesenteric angle and aortomesenteric distance can potentially cause compression of the third part of the duodenum, so called SMAS.

Factors that lead to an acute angulation of SMA can be either congenital or acquired. Congenital anomalies such as short Treitz ligament, malrotations and peritoneal adhesions have been described in association with this clinical condition. ${ }^{5,6}$ On the other hand, most common risk factors that cause a decrease in aortomesenteric angle are acquired and related to a loss of retroperitoneal fat following severe weight loss. Causes of excessive weight loss include but not limited to eating disorders, cachexia, malabsorption, polytrauma, malignancy and acquired immunodeficiency syndrome, large extensive burns, and major surgeries. ${ }^{7}$ Additionally, SMAS has been reported following orthopedic correction of spinal deformities, so called cast syndrome. ${ }^{8}$ According to previous studies, pronounced lumbar lordosis in females makes them more prone to develop this condition particularly in the age range from 10-39 years. ${ }^{6}$

Furthermore, acute pancreatitis complicated by SMAS is an extremely rare presentation, and only very few cases, describing this association, have been reported in the literature. ${ }^{9,10,11}$ This is the first report to describe SMAS in association with "recurrent" acute pancreatitis, to the best of our knowledge. In our patient, we believe that the recent excessive and rapid weight loss had resulted in a loss of retroperitoneal fat and, subsequently, a decrease in aortomesenteric angle, which lead ultimately to the duodenal compression. This was evident on the CT scan of the abdomen and pelvis, which was notable for the narrow aortomesenteric angle in addition to the compressed third part of duodenum leading to small bowel obstruction and dilation along with acute pancreatitis. The exact mechanism of pancreatitis in SMAS is still unknown, but it is proposed that the secondary occlusive postpapillary syndrome due to duodenal compression could cause reflux of bile into pancreas causing inflammation. On the other hand, inflammation of the pancreas could also cause paralytic ileus or peripancreatic edema thus reducing the aortomesenteric angle. ${ }^{9}$

Importantly, before SMAS diagnosis is made, other causes of GI obstruction should be ruled out, utilizing various imaging modalities. Traditionally, upper gastrointestinal barium swallow study has been used to diagnosis SMAS, which shows distended stomach and proximal duodenum along with abrupt vertical or oblique external compression of the third part of the duodenum. ${ }^{12}$ Also, conventional mesenteric angiography with hypotonic duodenography used to be the most efficient diagnostic tool for this syndrome in the past. ${ }^{12}$ Nevertheless, other noninvasive imaging modalities are more routinely utilized nowadays to diagnosis SMAS with high diagnostic accuracy including, multidetector CT scan, CT angiography and magnetic resonance angiography. ${ }^{4,13}$ These imaging techniques can accurately demonstrate duodenal obstruction due to SMA compression along with a narrow aortomesenteric angle and distance seen in this syndrome. ${ }^{13,14,15}$ Furthermore, in patients with proximal duodenum obstruction, an aortomesenteric angle of less than $22^{\circ}$ along with an aortomesenteric distance less than $8 \mathrm{~mm}$ on imaging corresponds strongly to SMAS. 7,16

As it was shown in our patient, SMAS complicated by acute pancreatitis is usually conservatively treated by correcting electrolyte disturbances, gastroduodenal decompression and nutritional support, with an ultimate goal of weight gain to allow for retroperitoneal fat pad reinforcement. ${ }^{5}$ Surgery is indicated for patients where conservative management fails. ${ }^{18}$ Available surgical options include gastro jejunostomy, duodenojejunostomy and lysis of the ligament of Treitz with mobilization of the duodenum. ${ }^{19,20}$ Duodenojejunostomy is the 
preferred surgical approach due to lower rates of surgical complications in addition to lower failure rate. ${ }^{21}$

\section{Conclusion}

Timely diagnosis and treatment of SMAS is pivotal to avoid serious complications such as fatal electrolyte abnormalities, gastric rupture, gut perforation, upper gastrointestinal bleeding, hypovolemic shock, oliguria, or sudden cardiovascular collapse. ${ }^{17}$. Since recurrent pancreatitis due to SMAS is a rare condition, increased awareness of its existence to facilitate early diagnosis and prompt treatment that is paramount.

\section{Conflict of Interest}

No conflict of interest to declare.

\section{Author Contribution Statement}

Rajshri Joshi: Conceptualization, Investigation, Writing-Original draft, Methodology, Resources, Visualization, Supervision. Zafar Alzubi: Editing, Figure analysis, Correspondence. Mo'tasem Alkhayyat: Figure analysis. Amar Gupta: Editing, Figure analysis. Daniel Mauro: Editing.

\section{References}

1. Wilkie DPD. Chronic duodenal ileus. Br J Surg . 1921;9(34):204-214. doi:10.1002/bjs.1800093405

2. Makary MS, Patel A, Aquino AM, Chamarthi SK. Clinical and Radiologic Considerations for Idiopathic Superior Mesenteric Artery Syndrome.Cureus . 2017;9(11). doi:10.7759/cureus.1822

3. Lamba R, Tanner DT, Sekhon S, McGahan JP, Corwin MT, Lall CG. Multidetector CT of vascular compression syndromes in the abdomen and pelvis. Radiographics . 2014;34(1):93-115. doi:10.1148/rg.341125010

4. Lippl F, Hannig C, Weiß W, Allescher HD, Classen M, Kurjak M. Superior mesenteric artery syndrome: Diagnosis and treatment from the gastroenterologist's view. J Gastroenterol . 2002;37(8):640-643. doi:10.1007/s005350200101

5. Suhani, Aggarwal L, Ali S, Jhaketiya A, Thomas S. Short and hypertrophic ligament of treitz: A rare cause of superior mesentric artery syndrome. J Clin Diagnostic Res . 2014;8(10):ND03-ND04. doi:10.7860/JCDR/2014/8852.4938

6. Akin JT, Skandalakis JE, Gray SW. The anatomic basis of vascular compression of the duodenum. Surg Clin North Am . 1974;54(6):1361-1370. doi:10.1016/S0039-6109(16)40491-3

7. Merrett ND, Wilson RB, Cosman P, Biankin A V. Superior mesenteric artery syndrome: Diagnosis and treatment strategies. J Gastrointest Surg . 2009;13(2):287-292. doi:10.1007/s11605-008-0695-4

8. Sapkas G, O'Brien JP. Vascular compression of the duodenum (cast syndrome) associated with the treatment of spinal deformities - A report of six cases. Arch Orthop Trauma Surg . 1981;98(1):7-11. doi:10.1007/BF00389703

9. Sihuay-Diburga DJ, Accarino-Garaventa A, Vilaseca-Montplet J, Azpiroz-Vidaur F. Acute pancreatitis and superior mesenteric artery syndrome. Rev Española Enfermedades Dig . 2013;105(10):626-628. doi:10.4321/s1130-01082013001000009

10. Gwee K, Teh A, Huang C. Acute superior mesenteric artery syndrome and pancreatitis in anorexia nervosa. Australas Psychiatry . 2010;18(6):523-526. doi:10.3109/10398562.2010.498885

11. Petrosyan M, Estrada JJ, Giuliani S, Williams M, Rosen H, Mason RJ. Gastric Perforation and Pancreatitis Manifesting after an Inadvertent Nissen Fundoplication in a Patient with Superior Mesenteric Artery Syndrome. Case Rep Med . 2009;2009:1-4. doi:10.1155/2009/426162

12. Gustafsson L, Falk A, Lukes PJ, Gamklou R. Diagnosis and treatment of superior mesenteric artery syndrome. Br J Surg . 1984;71(7):499-501. doi:10.1002/bjs.1800710706 
13. Agrawal GA, Johnson PT, Fishman EK. Multidetector row CT of superior mesenteric artery syndrome. J Clin Gastroenterol . 2007;41(1):62-65. doi:10.1097/MCG.0b013e31802dee64

14. Konen E, Amitai M, Apter S, et al. CT angiography of superior mesenteric artery syndrome. Am J Roentgenol . 1998;171(5):1279-1281. doi:10.2214/ajr.171.5.9798861

15. Welsch T, Büchler MW, Kienle P. Recalling superior mesenteric artery syndrome. Dig Surg . 2007;24(3):149-156. doi:10.1159/000102097

16. Kane KE, Koons AL. The Aortomesenteric Angle as an Aid in Diagnosing Superior Mesenteric Artery Syndrome. Clin Pract Cases Emerg Med . 2017;1(2):140-141. doi:10.5811/cpcem.2016.12.30676

17. Kabashi-Muqaj S, Kotori V, Lascu LC, Bondari S, Ahmetgjekaj I. The Role of MSCT in Superior Mesenteric Artery Syndrome (SMAS). Curr Heal Sci J . 2016;42(3):298-300. doi:10.12865/CHSJ.42.03.11

18. Ahmed AR, Taylor I. Superior mesenteric artery syndrome.Postgrad Med J . 1997;73(866):776-778. doi:10.1136/pgmj.73.866.776

19. Surgical D, Upon A. Vol. 148. 1958;148(5).

20. Gersin KS, Heniford BT. Laparoscopic duodenojejunostomy for treatment of superior mesenteric artery syndrome. JSLS . 1998;2(3):281-284. doi:10.1016/j.cireng.2013.06.003

21. Sun Z, Rodriguez J, McMichael J, et al. Minimally invasive duodenojejunostomy for superior mesenteric artery syndrome: a case series and review of the literature. Surg Endosc . 2015;29(5):1137-1144. doi:10.1007/s00464-014-3775-4 


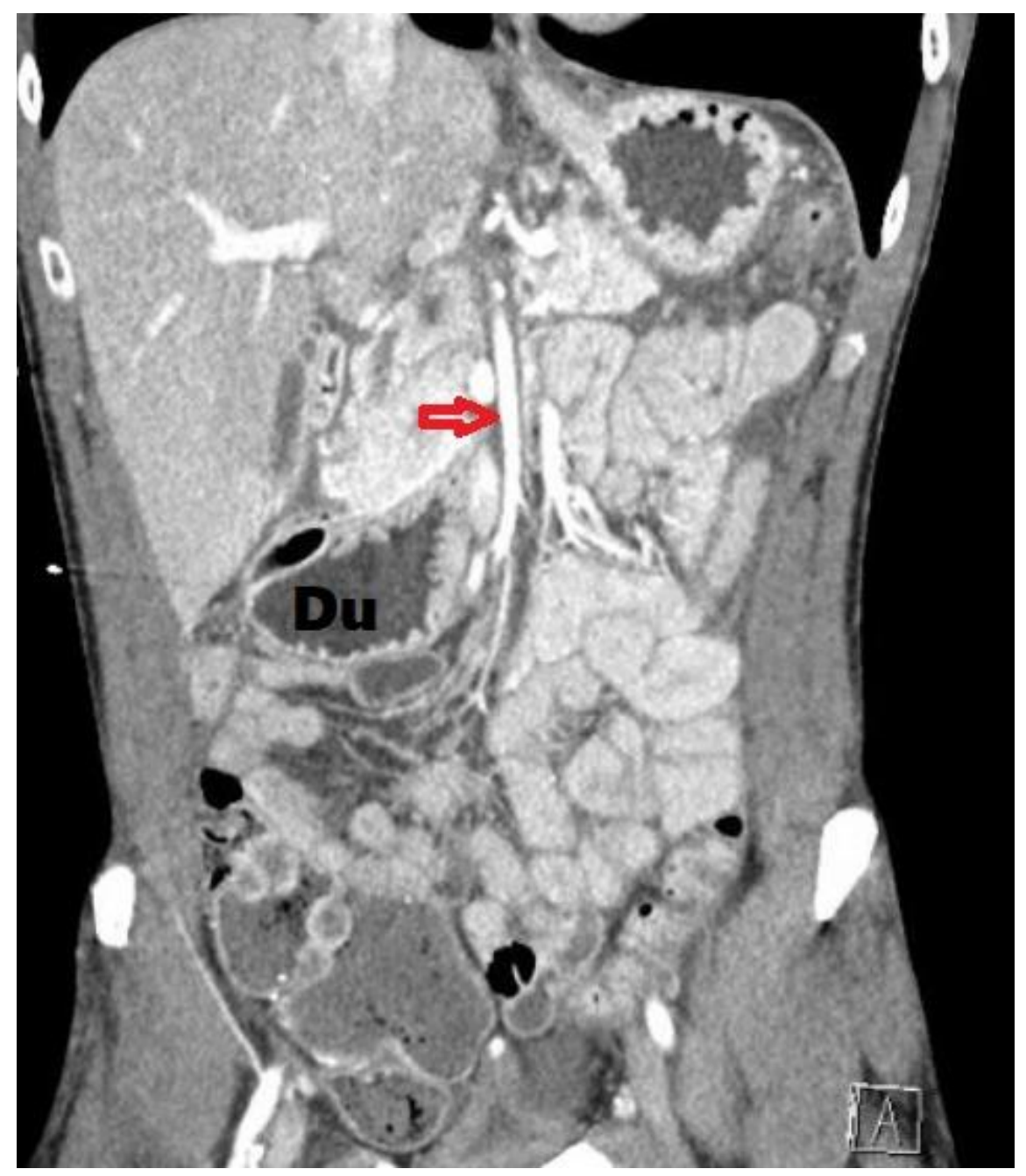



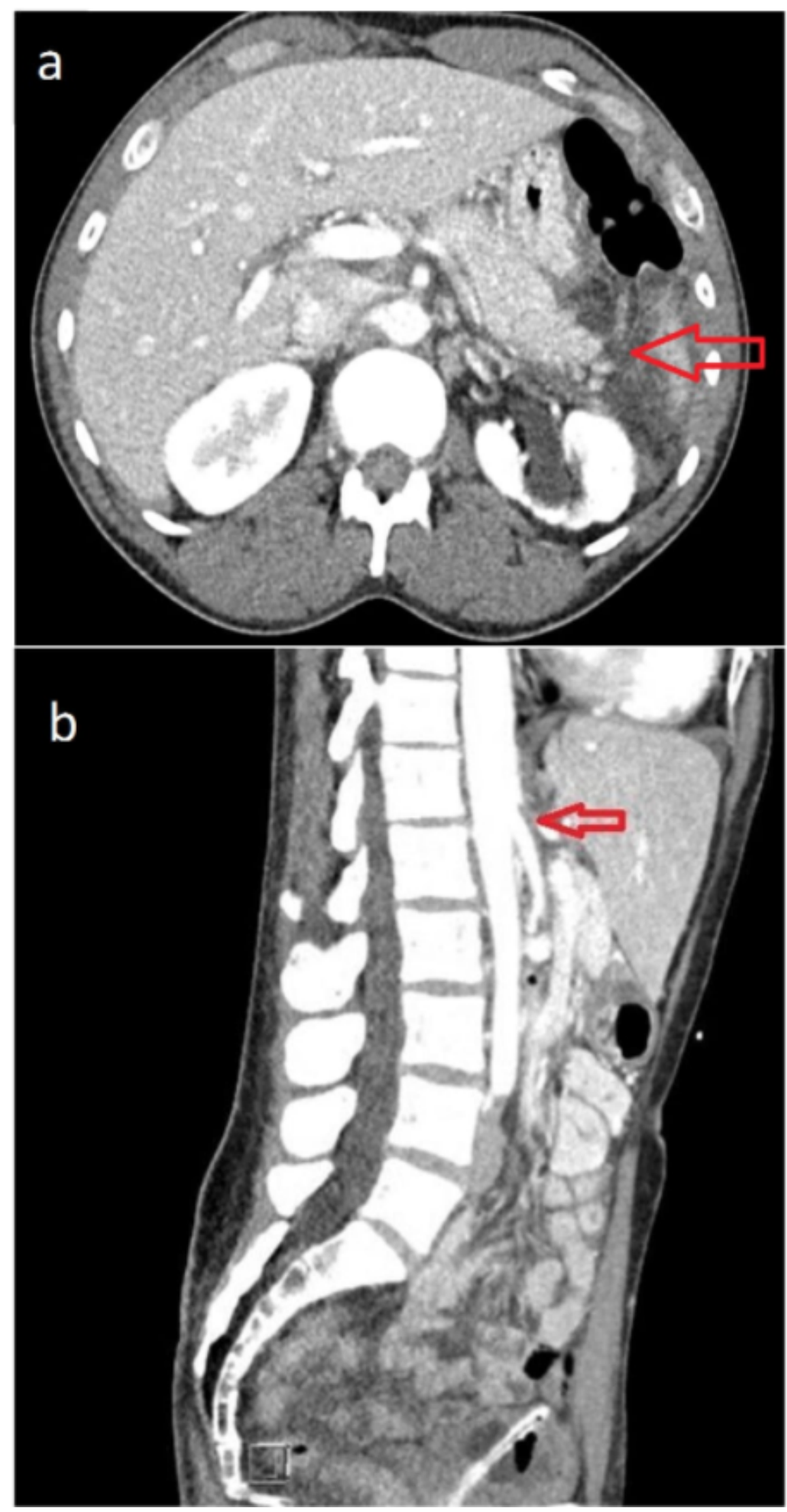


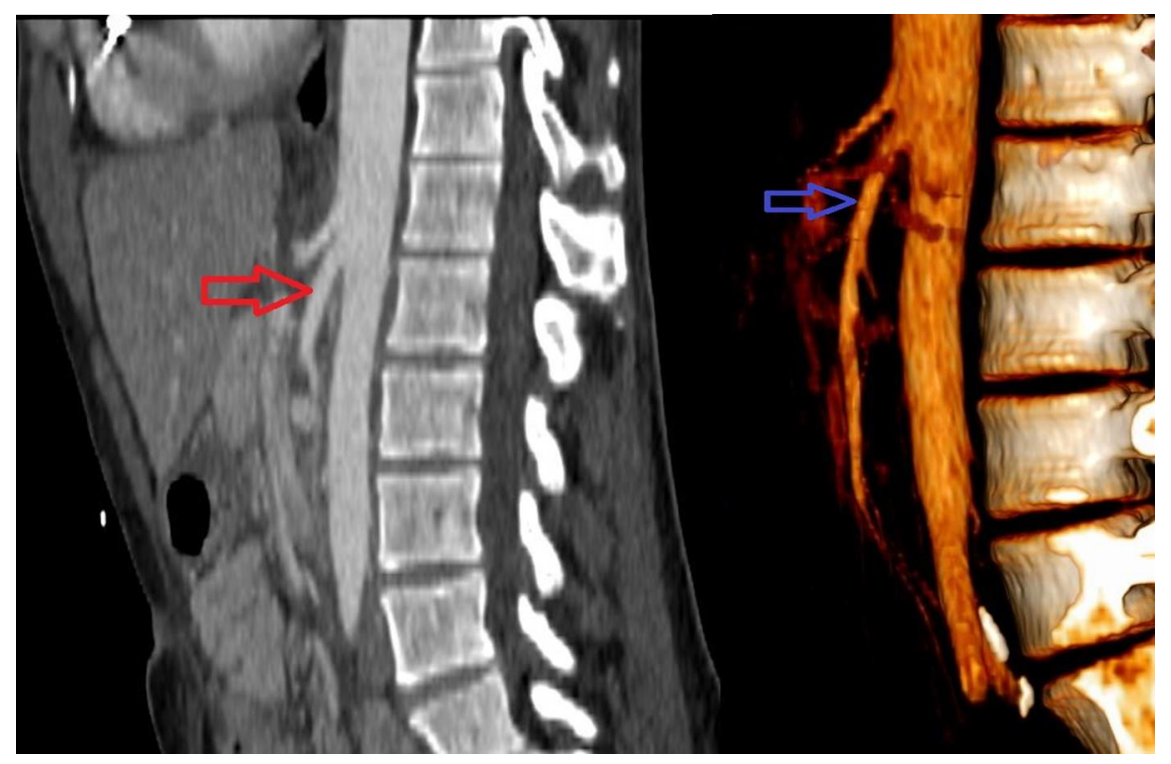

